\title{
A Two-Step Baro-Mechanical Cycle for Repeated Activation and Deactivation of Mechanophores
}

\author{
Sourabh Kumar ${ }^{1}$, Felix Zeller ${ }^{1}$ and Tim Stauch ${ }^{* 1,2,3}$ \\ 1 University of Bremen, Institute for Physical and Theoretical Chemistry, Leobener Straße NW2, D-28359 Bremen, Germany \\ 2 Bremen Center for Computational Materials Science, University of Bremen, Am Fallturm 1, D-28359 Bremen, Germany \\ ${ }^{3}$ MAPEX Center for Materials and Processes, University of Bremen, Bibliothekstraße 1, D-28359 Bremen, Germany
}

\begin{abstract}
Mechanophores that are embedded in a polymer backbone respond to the application of mechanical stretching forces by geometric changes such as bond rupture. Typically, these structural changes are irreversible, which limits the applicability of functional materials that incorporate mechanophores. Using computational methods, we here present a general method of restoring a force-activated mechanophore to its deactivated form by using hydrostatic pressure. We use the well-known spiropyran-merocyanine (SP-MC) interconversion to show that repeated activation and deactivation of the SP mechanophore can be achieved by alternating application of mechanical stretching forces and hydrostatic compression. In the baro-mechanical cycle, MC plays the role of a "barophore" that responds to hydrostatic pressure by bond formation. The activation and deactivation pathways of SP and MC are understood in terms of strain and electronic effects. Beneficially, this two-step baro-mechanical cycle can be observed in real time by using UV/Vis spectroscopy. Our calculations pave the way for massively improving the applicability and reusability of force-responsive materials.
\end{abstract}

\footnotetext{
* Corresponding author. Email: tstauch@uni-bremen.de
} 
Within the past two decades, mechanical force has emerged as a sustainable alternative to more traditional means of activating chemical reactions. ${ }^{[1,2]}$ At the focal point of many experimental and computational mechanochemical studies are mechanophores, i.e., small molecular units that are typically incorporated into the backbones of polymers. ${ }^{[3-5]}$ Mechanochemical deformation triggers profound structural rearrangements in the mechanophore, e.g. bond rupture. This effect has been used in the development of force-responsive and stress-sensing materials ${ }^{[6,7]}$ as well as for triggering reaction cascades ${ }^{[8,9]}$ and the release of small molecules. ${ }^{[10,11]}$ However, forceinduced structural changes in mechanophores are typically permanent. While some reversible mechanophores have been reported, ${ }^{[12]}$ the fact that most mechanophores cannot easily be reverted back to their deactivated form limits the applicability of force-responsive materials in everyday applications due to their limited recyclability.

Here we present a general approach to restore activated mechanophores to their original, deactivated form by applying hydrostatic pressure. Our proposed two-step baro-mechanical cycle is based on alternating application of mechanical stretching forces and hydrostatic pressure to switch between the activated and deactivated forms of mechanophores. In this computational study we focus on the well-known spiropyran (SP) mechanophore, which can be transformed to merocyanine (MC) by application of mechanical stretching forces. ${ }^{[6,13]}$ (Figure 1). While the reverse reaction of $\mathrm{MC}$ to SP has been initiated by using light, ${ }^{[14,15]}$ heat, ${ }^{[16]}$ guest molecules ${ }^{[17]}$ and redox reactions, ${ }^{[18]}$ we suggest that our method of restoring a mechanophore to its deactivated form by applying hydrostatic pressure is generally applicable to mechanophores that experience an increase in volume upon activation. Beneficially, the interconversion of SP and MC can be tracked by changes in the visible part of the UV/Vis absorption spectrum.

To demonstrate the gist of the baro-mechanical cycle, stretching forces were applied to the ends of short linkers attached to SP (Figure 1) with the External Force is Explicitly Included $(E F E I)^{[19-21]}$ method. At the B3LYP ${ }^{[22-24]} / 6-31 G(d)^{[25]}$ level of theory, a force of $2.7 \mathrm{nN}$ is required to initiate the isomerization from SP to MC. Conversely, application of a hydrostatic pressure of $5 \mathrm{GPa}$ via the eXtended Hydrostatic Compression Force Field (X-HCFF) ${ }^{[26]}$ to MC leads to the generation of SP. In this case, MC acts as a "barophore", in analogy to the role of SP as a mechanophore. Computational details can be found in the Supporting Information.

While these results suggest the possibility of returning force-activated mechanophores to their deactivated state by applying hydrostatic pressure, it is crucial to note that compression is a common method of activating mechanophores ${ }^{[27-29]}$ and not of deactivating them. However, if the activated form of a mechanophore has a higher volume than the deactivated form, the application of hydrostatic pressure clearly favors the formation of the deactivated form. In the 


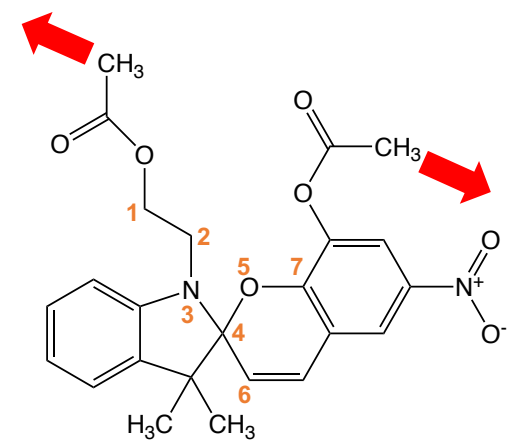

Spiropyran (SP)
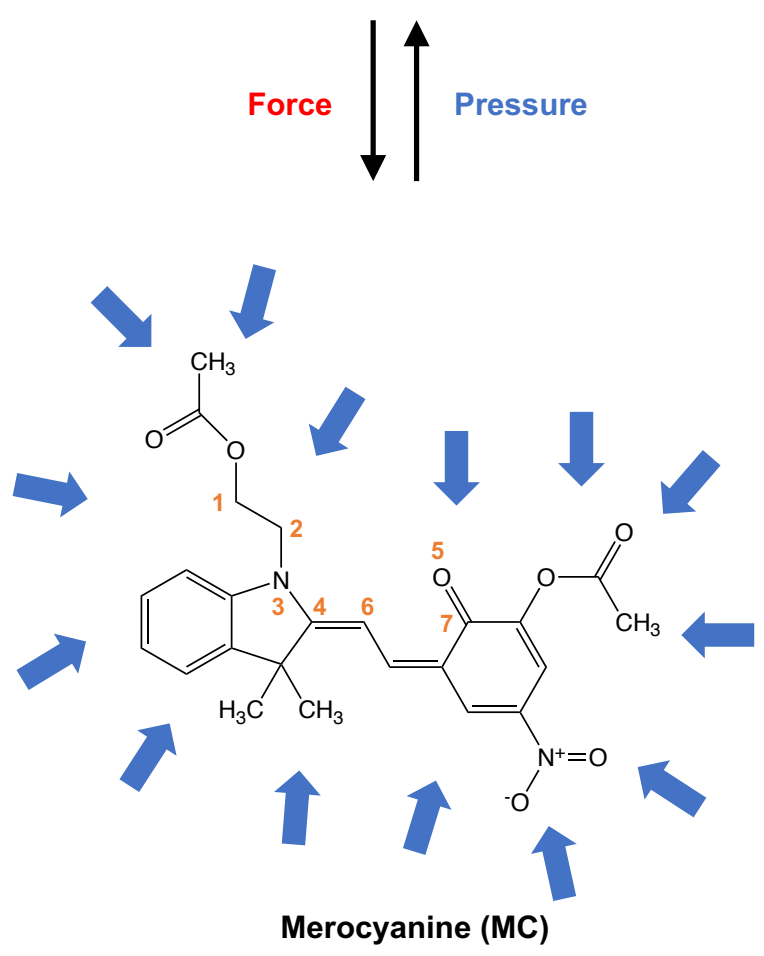

Figure 1: Schematic illustration of the two-step baro-mechanical cycle, in which the isomerization of spiropyran (SP) to merocyanine (MC) is triggered by mechanical stretching forces, whereas the opposite transformation is initiated by hydrostatic pressure.

case of SP-MC isomerism, we have calculated the volume of SP to be $352.8 \AA^{3}$ and that of MC to be $357.7 \AA^{3}$ (both molecules without linkers), based on electron densities. Since the chemical equilibrium can be biased towards species with a lower volume by application of hydrostatic pressure, ${ }^{[30-32]}$ we come to the conclusion that mechanical compression in experiments is usually not hydrostatic, which has been indicated before in the case of the dianthracene mechanophore. ${ }^{[33]}$ At the single-molecule level, compression is likely anisotropic, owing, e.g., to steric effects of the (polymer) environment. If, however, truly hydrostatic compression was achieved experimentally at the single-molecule level, we expect efficient deactivation of force-activated mechanophores to be possible. 


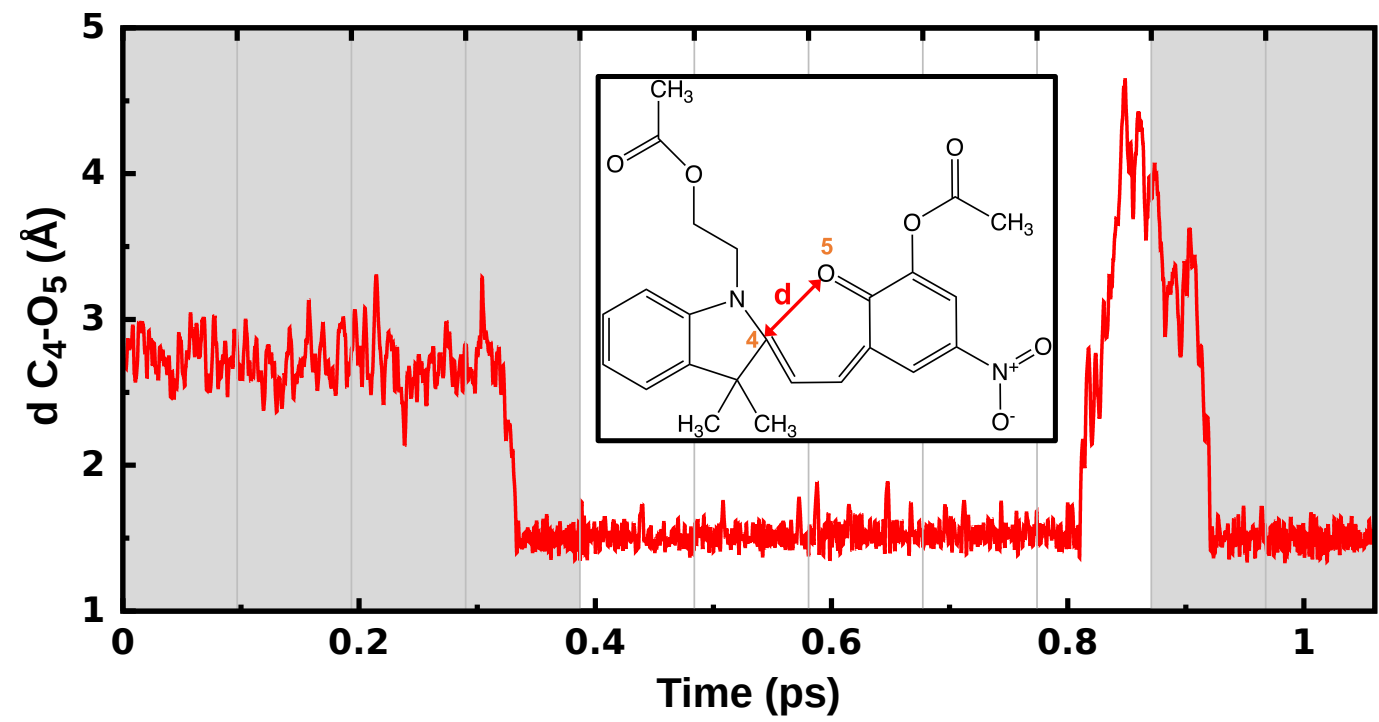

Figure 2: Temporal progress of the $\mathrm{C}_{4}-\mathrm{O}_{5}$ bond (cf. Figure 1 for the numbering scheme), which ruptures during isomerization of SP to MC and forms during the reverse process, throughout the course of a representative Born-Oppenheimer Molecular Dynamics (BOMD) trajectory. Pressure was increased in steps of $0.5 \mathrm{GPa}$ throughout the gray shaded areas. Stretching forces were increased in steps of $300 \mathrm{pN}$ in the white area. Gray vertical lines signify the points in time when the pressure or the force were adjusted, respectively.

To gain a dynamic picture of the repeated activation/deactivation process of the SP mechanophore, ten Born-Oppenheimer Molecular Dynamics (BOMD) simulations at a temperature of $1000 \mathrm{~K}$ were conducted. This temperature was adjusted to sample a considerable part of the conformational space within a reasonable computation time. Starting from MC, a step-wise increasing pressure was applied until SP was formed. In a representative BOMD trajectory (Figure 2), a pressure of $2 \mathrm{GPa}$ was sufficient to form the $\mathrm{C}_{4}-\mathrm{O}_{5}$ bond at approx. $300 \mathrm{fs}$ simulation time (cf. Figure 1 for the numbering scheme), leading to SP. Subsequently, pressure was switched off and an increasing force was applied. At approx. 800 fs simulation time and a stretching force of $750 \mathrm{pN}$ applied to $\mathrm{SP}$, the $\mathrm{C}_{4}-\mathrm{O}_{5}$ bond is ruptured and $\mathrm{MC}$ is formed. The interconversion between SP and MC also becomes apparent in the decrease in double bond character of the $\mathrm{C}_{7}-\mathrm{O}_{5}$ bond (Figure $\mathrm{S} 1$ ).

To demonstrate the reversibility of this process, pressure was subsequently applied once more. This time, a pressure of $0.5 \mathrm{GPa}$ was sufficient to transform SP to MC, which is due to thermal oscillations in combination with the proximity of $\mathrm{C}_{4}$ and $\mathrm{O}_{5}$. In another trajectory, stretching forces applied to SP generated a fully extended, "trans" configuration of MC with a calculated volume of $358.8 \AA^{3}$, which required a significantly higher pressure (3.5 GPa) to trigger the transformation to SP. Hence, the pressure required for deactivation depends heavily on the instantaneous configuration of MC. Details on the required pressures and forces in all other BOMD trajectories are given in the Supporting Information (Table S1).

The force-induced activation of SP in the representative BOMD trajectory discussed above 

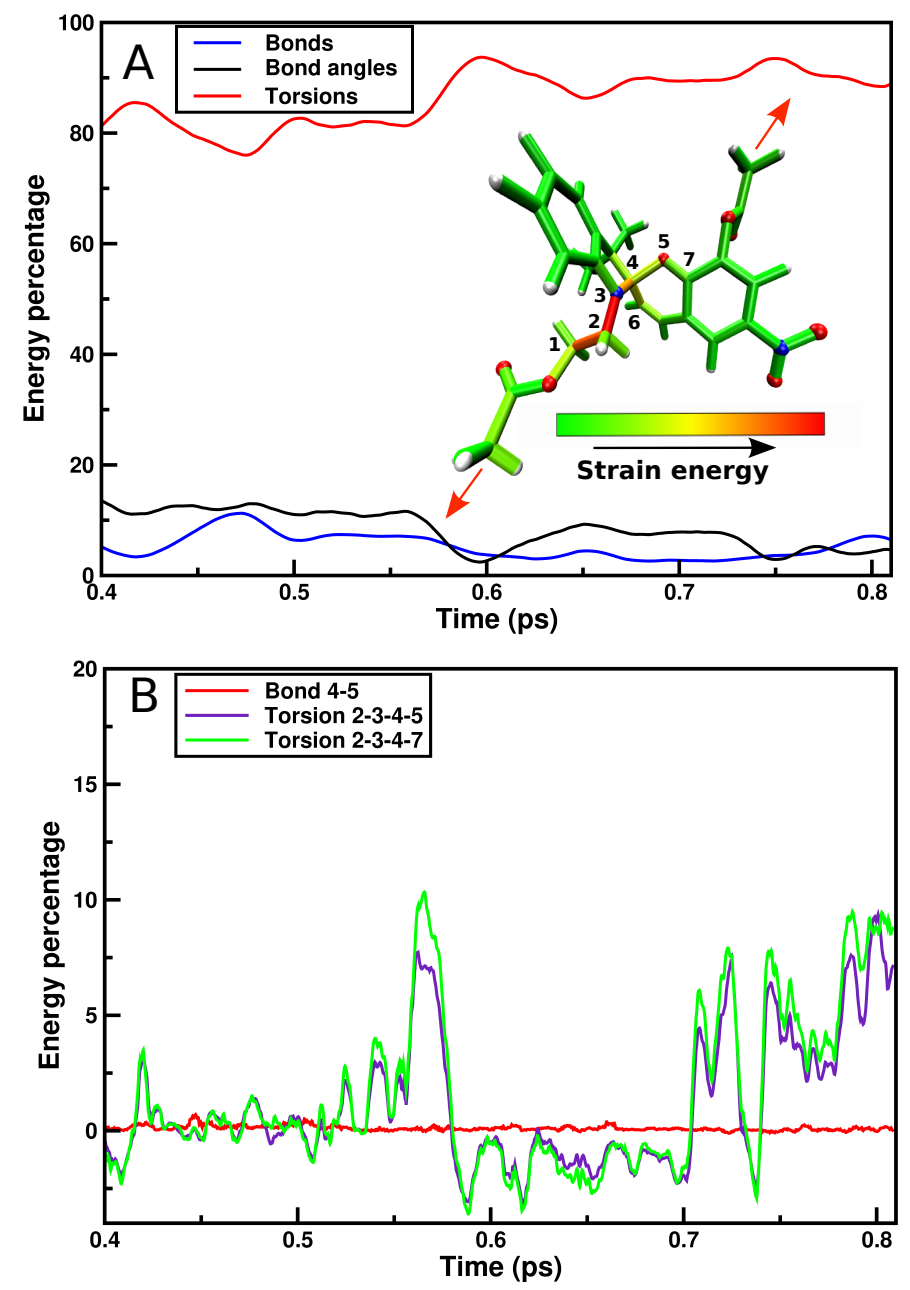

Figure 3: Results of the Judgement of Energy DIstribution (JEDI) analysis for SP activation during a representative BOMD trajectory. A) Percentages of strain energy in the bonds, bendings and torsions of stretched SP after it is formed initially. The color-coded structure is a representation of stretched SP shortly before rupture, at an external force of $750 \mathrm{pN}$ at $814 \mathrm{fs}$ simulation time. Strain energies in all degrees of freedom were mapped onto the bonds. B) Percentages of strain energy in the scissile bond and in two dihedral angles.

was analyzed in terms of strain energies by applying the Judgement of Energy Dlstribution (JEDI) analysis. ${ }^{\left[{ }^{34]}\right.}$ As shown in Figure 3, surprisingly, only a very small percentage of strain is stored in the scissile $\mathrm{C}_{4}-\mathrm{O}_{5}$ bond when stretching forces are applied. Instead, torsions in the vicinity of the scissile bond are significantly displaced, leading to an accumulation of strain energy in these torsions. A similar effect has been observed before in knotted polymer strands, where accumulation of strain in torsions facilitates bond rupture. ${ }^{[35]}$ The facilitation of bond rupture in SP by torsional motions in the vicinity of the scissile bond has, to the best of our knowledge, never been observed before. However, we assume that this effect explains the experimentally observed high mechanical yield of the SP-to-MC conversion.

The interconversion between SP and MC can be observed via time-resolved changes in the UV/Vis absorption spectrum (Figure 4). Initially, peaks in the range between 400 and $600 \mathrm{~nm}$ are 


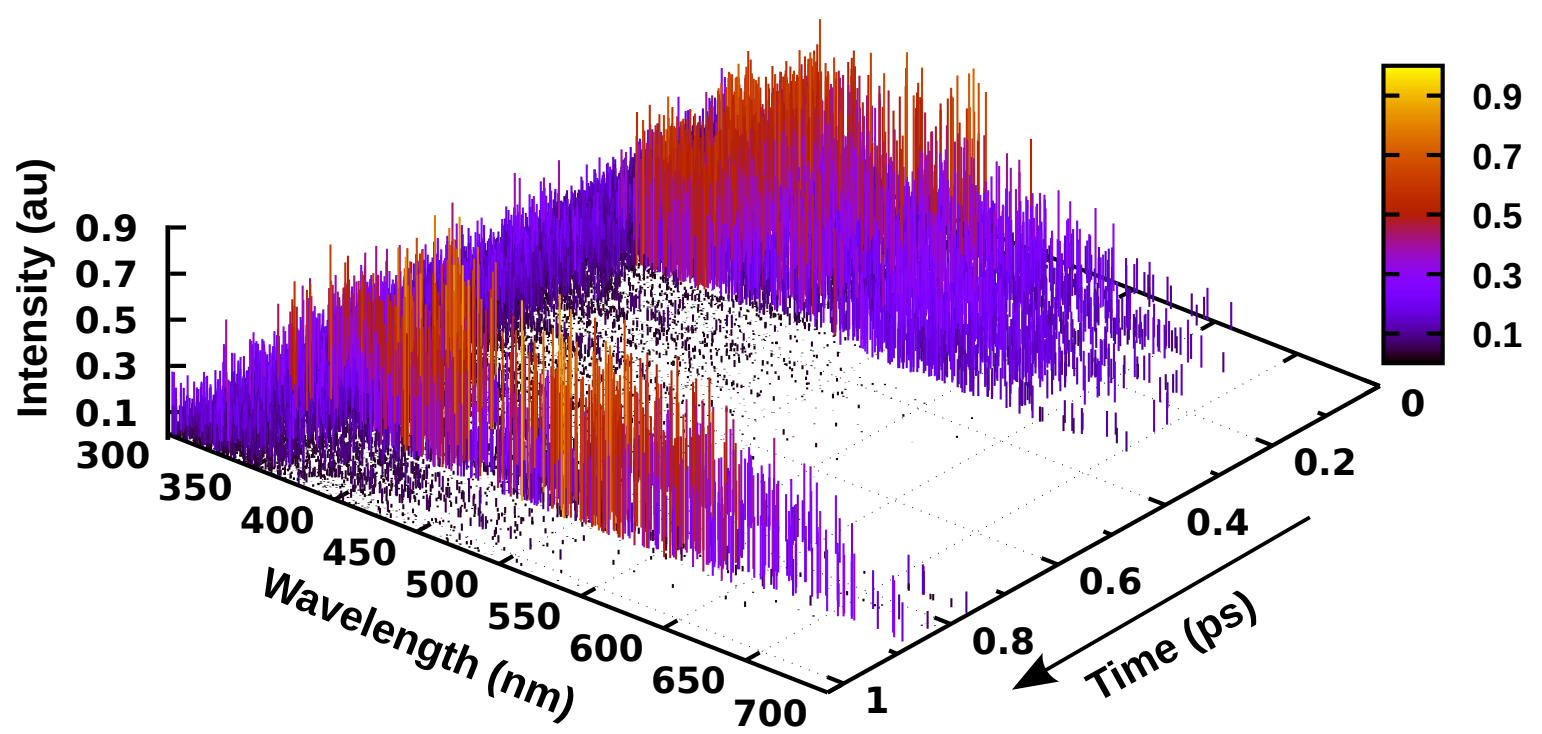

Figure 4: Time series plot of the UV absorption spectrum of the SP/MC system under the alternating influences of pressure and force throughout a representative BOMD trajectory. A color palette is used to highlight the intensities.

found due to the presence of MC. Initially, the absorption spectrum remains relatively constant as pressure increases. However, as soon as SP is formed, peaks in the UV/Vis absorption spectrum are limited to the range between 300 and $350 \mathrm{~nm}$, signifying the characteristic color change that is observed when switching between MC and SP. The absorption spectrum of SP is relatively insensitive to the application of stretching forces until MC is formed, where the initial absorption spectrum is recovered. The final generation of SP by pressure is again accompanied by a marked blue shift of the absorption spectrum.

The marked changes in the absorption spectrum when switching between SP and MC are useful for experimental verification of the baro-mechanical cycle presented here, since the SP-MC interconversion can be tracked in real time either spectroscopically or with the naked eye. Irrespective of the changes in UV/Vis absorption, however, we assume that force-activated mechanophores can act as barophores when their volume is higher than that of the original, deactivated mechanophore.

By using static and dynamic calculations of the spiropyran-merocyanine (SP-MC) system under alternating influences of mechanical stretching forces and hydrostatic pressure, a twostep baro-mechanical cycle for repeated activation and deactivation of mechanophores was established. The activation of SP was understood in terms of strain and it was found that SP is "twisted open" by force, which greatly facilitates bond rupture. Conversely, MC acts as a barophore that responds to the application of pressure by bond formation. Marked changes in the visible part of the UV/Vis absorption spectrum allow the real-time tracking of the SP-MC interconversion. We hope that our proof-of-principle study triggers the interest in 
combined mechanochemical and barochemical experiments, using, e.g., diamond-anvil cells and ultrasound baths. The possibility to restore force-activated mechanophores to their deactivated form holds great promise for the development of reusable force-responsive materials. In addition, the color change associated with the MC-to-SP conversion paves the way for the use of MC as an internal standard in high-pressure experiments. Calculations on molecular pressure sensors are currently being conducted in our group.

Acknowledgements: The authors would like to thank M. Scheurer, University of Heidelberg, for providing the python script to calculate the volume of a molecule. 


\section{References}

[1] S. L. James, C. J. Adams, C. Bolm, D. Braga, P. Collier, T. Friščić, F. Grepioni, K. D. Harris, G. Hyett, W. Jones, A. Krebs, J. Mack, L. Maini, A. G. Orpen, I. P. Parkin, W. C. Shearouse, J. W. Steed, and D. C. Waddell, "Mechanochemistry: opportunities for new and cleaner synthesis," Chem. Soc. Rev., vol. 41, pp. 413-447, 2012.

[2] K. J. Ardila-Fierro and J. G. Hernández, "Sustainability Assessment of Mechanochemistry by Using the Twelve Principles of Green Chemistry," ChemSusChem, vol. 14, pp. 2145-2162, 2021.

[3] I. M. Klein, C. C. Husic, D. P. Kovács, N. J. Choquette, and M. J. Robb, "Validation of the CoGEF Method as a Predictive Tool for Polymer Mechanochemistry," J. Am. Chem. Soc., vol. 142, pp. 16364-16381, 2020.

[4] G. De Bo, "Polymer Mechanochemistry and the Emergence of the Mechanophore Concept," Macromolecules, vol. 53, pp. 7615-7617, 2020.

[5] C. E. Diesendruck, "Mechanophores for Chemical Function," in Mechanochemistry in Materials (Y. C. Simon and S. L. Craig, eds.), ch. 2, pp. 36-52, The Royal Society of Chemistry, 2018.

[6] H. Traeger, D. J. Kiebala, C. Weder, and S. Schrettl, "From Molecules to Polymers - Harnessing Inter- and Intramolecular Interactions to Create Mechanochromic Materials," Macromol. Rapid Commun., vol. 42, p. 2000573, 2021.

[7] R. Göstl, J. M. Clough, and R. P. Sijbesma, "Optical Sensing of Stress in Polymers," in Mechanochemistry in Materials (Y. C. Simon and S. L. Craig, eds.), ch. 3, pp. 53-75, The Royal Society of Chemistry, 2018.

[8] H. Hu, Z. Ma, and X. Jia, "Reaction Cascades in Polymer Mechanochemistry," Mater. Chem. Front., vol. 4, pp. 3115-3129, 2020.

[9] Z. Chen, X. Zhu, J. Yang, J. A. Mercer, N. Z. Burns, T. J. Martinez, and Y. Xia, "The cascade unzipping of ladderane reveals dynamic effects in mechanochemistry," Nat. Chem., vol. 12, pp. 302-309, 2020.

[10] L. J. Mier, G. Adam, S. Kumar, and T. Stauch, "The Mechanism of Flex-Activation in Mechanophores Revealed By Quantum Chemistry," ChemPhysChem, vol. 21, pp. 2402-2406, 2020.

[11] M. B. Larsen and A. J. Boydston, ""Flex-activated" mechanophores: Using polymer mechanochemistry to direct bond bending activation," J. Am. Chem. Soc., vol. 135, no. 22, pp. 8189-8192, 2013.

[12] T. Stauch, "Mechanical Switching of Aromaticity and Homoaromaticity in Molecular Optical Force Sensors for Polymers," Chem. Eur. J., vol. 24, pp. $7340-7344,2018$.

[13] D. A. Davis, A. Hamilton, J. Yang, L. D. Cremar, D. Van Gough, S. L. Potisek, M. T. Ong, P. V. Braun, T. J. Martínez, S. R. White, J. S. Moore, and N. R. Sottos, "Force-induced activation of covalent bonds in mechanoresponsive polymeric materials," Nature, vol. 459, pp. 68-72, may 2009.

[14] M. Ghani, A. Heiskanen, J. Kajtez, B. Rezaei, N. B. Larsen, P. Thomsen, A. Kristensen, A. Žukauskas, M. Alm, and J. Emnéus, "On-Demand Reversible UV-Triggered Interpenetrating Polymer Network-Based Drug Delivery System Using the SpiropyranMerocyanine Hydrophobicity Switch," ACS Appl. Mater. Interfaces, vol. 13, no. 3, pp. 3591-3604, 2021.

[15] T. Yamaguchi, K. J. Imwiset, and M. Ogawa, "Efficient Negative Photochromism by the Photoinduced Migration of Photochromic Merocyanine/Spiropyran in the Solid State," Langmuir, vol. 37, pp. 3702-3708, 2021.

[16] Z. Cao, "Highly Stretchable Tough Elastomers Crosslinked by Spiropyran Mechanophores for Strain-Induced Colorimetric Sensing," Macromol. Chem. Phys., vol. 221, no. 15, pp. 1-7, 2020.

[17] M. Li, L. Lei, Q. Zhang, and S. Zhu, "CO2-Breathing Induced Reversible Activation of Mechanophore within Microgels," Macromol. Rapid Commun., vol. 37, no. 12, pp. 957-962, 2016.

[18] J. D. Steen, D. R. Duijnstee, A. S. Sardjan, J. Martinelli, L. Kortekaas, D. Jacquemin, and W. R. Browne, "Electrochemical Ring-Opening and -Closing of a Spiropyran," J. Phys. Chem. A, vol. 125, pp. 3355-3361, 2021.

[19] M. T. Ong, J. Leiding, H. Tao, A. M. Virshup, and T. J. Martínez, "First Principles Dynamics and Minimum Energy Pathways for Mechanochemical Ring Opening of Cyclobutene.," J. Am Chem. Soc., vol. 131, pp. 6377-6379, 2009. 
[20] J. Ribas-Arino, M. Shiga, and D. Marx, "Understanding Covalent Mechanochemistry.," Angew. Chem. Int. Ed., vol. 48, pp. 4190-4193, 2009.

[21] K. Wolinski and J. Baker, "Theoretical predictions of enforced structural changes in molecules," Mol. Phys., vol. 107, no. 22, pp. 2403-2417, 2009.

[22] A. D. Becke, "Correlation energy of an inhomogeneous electron gas: A coordinate-space model," J. Chem. Phys., vol. 88, no. 2, pp. 1053-1062, 1988.

[23] C. Lee, W. Yang, and R. G. Parr, "Development of the Colic-Salvetti correlation-energy formula into a functional of the electron density," Phys. Rev. B, vol. 37, no. 2, pp. 785-789, 1988.

[24] A. D. Becke, "A new mixing of Hartree-Fock and local density-functional theories," J. Chem. Phys., vol. 98, no. 2, pp. 13721377, 1993.

[25] W. J. Hehre, R. Ditchfield, and J. A. Pople, "Self - Consistent Molecular Orbital Methods . XII . Further Extensions of Gaussian - Type Basis Sets for Use in Molecular Orbital Studies of Organic Molecules," J. Chem. Phys., vol. 56, pp. 22572261, 1972.

[26] T. Stauch, "A mechanochemical model for the simulation of molecules and molecular crystals under hydrostatic pressure," $J$. Chem. Phys., vol. 153, no. 13, p. 134503, 2020.

[27] X. Meng, G. Qi, C. Zhang, K. Wang, B. Zou, and Y. Ma, "Visible mechanochromic responses of spiropyrans in crystals via pressure-induced isomerization," Chem. Commun., vol. 51, pp. 9320-9323, 2015.

[28] X. Meng, G. Qi, X. Li, Z. Wang, K. Wang, B. Zou, and Y. Ma, "Spiropyran-based multi-colored switching tuned by pressure and mechanical grinding," J. Mater. Chem. C, vol. 4, no. 32, pp. 7584-7588, 2016.

[29] C. P. Kabb, C. S. O'Bryan, C. D. Morley, T. E. Angelini, and B. S. Sumerlin, "Anthracene-based mechanophores for compression-activated uorescence in polymeric networks†," Chem. Sci., vol. 10, pp. 7702-7708, 2019.

[30] S. Kumar and T. Stauch, "Trapping the Transition State in a [2, 3]-Sigmatropic Rearrangement by Applying Pressure," ChemRxiv, pp. DOI: 10.33774/chemrxiv-2021-7d2r7.

[31] T. Stauch, "Quantum chemical modeling of molecules under pressure," Int. J. Quantum Chem., vol. 121, no. 3, pp. 1-8, 2020.

[32] B. Chen, R. Hoffmann, and R. Cammi, "The Effect of Pressure on Organic Reactions in Fluids-a New Theoretical Perspective," Angew. Chem. Int. Ed., vol. 56, pp. 11126-11142, 2017.

[33] R. Fukuda and K. Nakatani, "Quantum Chemical Study on the High-Pressure Eect for [4+4] Retrocycloaddition of Anthracene Cyclophane Photodimer," J. Phys. Chem. C, vol. 123, p. 44934501, 2019.

[34] T. Stauch and A. Dreuw, "A quantitative quantum-chemical analysis tool for the distribution of mechanical force in molecules," J. Chem. Phys., vol. 140, no. 13, p. 134107, 2014.

[35] T. Stauch and A. Dreuw, "Knots "Choke Off" Polymers upon Stretching," Angew. Chem. Int. Ed, vol. 55, pp. 811-814, 2016. 